Die Datenübermittlung ist nur zulässig, wenn auf beiden Stufen ein positives Prüfungsergebnis vorliegt.

\section{Hochsicherheitshandy der Telekom erhält BSI-Zulassung}

Das Security-Smartphone SiMKo 3 der Telekom hat die Prüfung durch das Bundesamt für Sicherheit in der Informationstechnik erfolgreich abgeschlossen. Damit erhält die neue Generation der Hochsicherheitshandys offiziell die Zulassung für die Geheimhaltungsstufe VS-NfD (Verschlusssache - Nur für den Dienstgebrauch). Mitgliedern der Bundesregierung sowie Mitarbeitern von Ministerien und Bundesbehörden steht für besonders vertrauliche Nachrichten damit künftig erstmals ein Mobilgerät zur Verfügung, das den neu entwickelten L4-Hochsicherheits-Mikrokern als Betriebssystem in sich trägt.

Der Kern hat nur wenige 10.000 Zeilen Programmcode, handelsübliche Smartphones haben dagegen Millionen Zeilen. Stephan Maihoff, bei der Telekom für SiMKo verantwortlich, sagt: „So große Betriebssysteme, die sich auch noch sehr schnell weiterentwickeln, sind praktisch nicht prüfbar. Hintertüren lassen sich da nicht ausschließen. Gegen das Hacker-Risiko setzen wir einen transparenten Kern, der kein Versteck für Überraschungen und Sicherheit von innen bietet."

Mikrokern und Sicherheitstechnik kommen aus Deutschland. Bei Kern und Sicherheitstechnik des SiMKo 3 setzt die Telekom durchgängig auf Unternehmen aus Deutschland. So kommt die Kryptokarte von certgate, für verschlüsselte Verbindungen sorgt NCP beides Unternehmen aus Nürnberg. Das L4-Mikrokern-System haben die TU Dresden, das Dresdener Startup Kernkonzept, die Telekom Innovation Laboratories sowie das Berliner Startup Trust2Core entwickelt.

Samsung will sicheres Telefonieren und Surfen vorantreiben. Möglich wurde die Implementierung des Kerns nur durch die besonders enge Zusammenarbeit mit Samsung. Durch die Kooperation des SiMKo 3-Projektteams und der Samsung-Entwicklungsabteilung wurde gemeinsam ein Hochsicherheits-Smartphone auf Basis des GALAXY SIII auf den Markt gebracht.

\section{Consumerization of IT, Studie von techconsult: Arbeitnehmer wollen Takt in der Unternehmens IT mitbestimmen}

Immer mehr Mitarbeiter in deutschen Unternehmen projizieren private IT-Gewohnheiten auf den Arbeitsplatz - und dieser Trend steigt rasant an. Das geht aus dem am 20.09.2013 von Microsoft veröffentlichten Market Paper "Consumerization Study CIO Challenges - Consumer-orientierte IT im Wandel der Zeit" hervor, das techconsult im Auftrag von Microsoft verfasst hat.

Waren es zu Beginn der Erhebung im Oktober 2012 noch 69 Prozent der Arbeitnehmer, die private Endgeräte auch im beruflichen Alltag nutzen, sind es aktuell bereits 81 Prozent - ein Anstieg von mehr als 17 Prozent.

Auch der Einsatzgrad privater Anwendungen hat sich nahezu verdoppelt. Während aktuell 41 Prozent der befragten Arbeitneh- mer private Anwendungen auch im Beruf nutzen, waren es im Oktober 2012 erst 26 Prozent.

"Bring your own" - Unternehmen stellen sich dem Trend Nicht nur bei Arbeitnehmern ist ein signifikanter Anstieg bei der Umsetzung von Consumerization of IT zu verzeichnen. So öffnen sich immer mehr Unternehmen für "Bring your own" und ermöglichen ihren Mitarbeitern, sich auch im beruflichen Alltag in ihrem gewohnten bzw. gewünschten technologischen Kommunikationsund Arbeitsumfeld zu bewegen.

Diese Einschätzung bestätigt auch techconsult. Während zu Beginn der Studie im Oktober 2012 nur 27 Prozent der befragten Mitarbeiter angaben, dass ihr Unternehmen den Einsatz von privaten Endgeräten befürwortet, sind es aktuell 41 Prozent und damit nahezu doppelt so viele. "IT ist heute Teil unserer Identität, die uns im Alltag - privat wie beruflich - begleitet", sagt Thomas Schröder, Geschäftsführer Großkunden und Partner, Microsoft Deutschland $\mathrm{GmbH}$. „Arbeitnehmer, gerade jene aus der Generation Y, lassen sich die Freiheit, mit den Geräten und Lösungen ihrer Wahl zu arbeiten, nicht mehr nehmen“, so Schröder weiter, „,Arbeitgeber profitieren gleichzeitig von höherer Motivation und Produktivität der Mitarbeiter, wenn sie eine sichere und nahtlose Einbindung der Geräte in die Unternehmens IT gewähren."

\section{Endgerät ist nicht gleich Endgerät}

Die Ergebnisse zeigen auch, dass sich der Einsatzgrad der einzelnen Geräte signifikant verändert hat. Vor allem Smartphones sind ein wichtiger Begleiter im privaten Alltag, auf den auch beruflich kaum jemand mehr verzichten möchte. So stieg der Anteil privater Smartphones im Unternehmensalltag um sieben auf insgesamt 74 Prozent. Im Gegensatz dazu ist der Anteil der herkömmlichen Mobiltelefone auf 24 Prozent gesunken.

Den größten Sprung machten jedoch die privaten Tablet-PCs. Ihr Einsatzgrad verdoppelte sich nahezu in einem halben Jahr und liegt jetzt bei 29 Prozent.

\section{World Quality Report: Rund ein Viertel der IT-Budgets fließt in Qualitätssicherung und Software-Testing}

Berlin, 12. September 2013 - Die Ausgaben für Software-Testing und Qualitätssicherung (QS) machen mittlerweile 23 Prozent der weltweiten IT-Budgets aus - so ein Ergebnis des am 12.09.2013 veröffentlichten World Quality Reports von Capgemini, Sogeti und HP mit weltweit 1.500 Befragten. Der durchschnittliche Anteil am ITBudget stieg von 18 Prozent im Jahr 2012 auf aktuell knapp ein Viertel an. Vielen Organisationen fällt es allerdings immer noch schwer, den Mehrwert abzubilden, den sie durch die Testing-Funktionen erzielen.

\section{Deutschland führend im Qualitätsbewusstsein}

Für Deutschland zeichnen sich drei Trends ab: Aktuell investieren deutsche Organisationen 22 Prozent ihrer IT-Budgets in das Thema Qualitätssicherung, 2012 waren es noch 20 Prozent. Es wird erwartet, dass der Anteil in den kommenden zwei Jahren auf bis zu 26 Prozent anwachsen wird und Deutschland gemessen daran einer der weltweit reifsten sowie qualitätsorientiertesten Märkte bleibt. Bei Outsourcing und Managed-Testing-Services-Modellen hinge- 